\title{
wolfexplorer: a tool for visualization and exploration of complex multi-year multi-specimen datasets
}

\section{Roman Luštrik $^{1}$ and Žan Kuralt ${ }^{2}$}

1 Genialis, Inc. 2 Biotechnical faculty, University of Ljubljana, Jamnikarjeva 101, SI-1000 Ljubljana, Slovenia

DOI: $10.21105 /$ joss. 01064

\section{Software}

- Review ¿

- Repository ¿

- Archive ca

Submitted: 09 October 2018

Published: 10 December 2018

\section{License}

Authors of papers retain copyright and release the work under a Creative Commons Attribution 4.0 International License (CC-BY).

\section{Summary}

Non-invasive genetic sampling has become an accessible method for monitoring wild animal populations, whereas visualising such multi-specimen data in both spatial and temporal context has proven to be an arduous task. Conventional approach utilising GIS software suffers from a lack of interactivity, particularly when analysing many samples from numerous animals simultaneously. Yet such datasets, if disentangled properly, offer an invaluable insight into movement patterns, habitat utilization and life histories of studied animals.

Here we present wolfexplorer - a tool for visualization and exploration of complex multiyear multi-specimen datasets. Package, incorporating user-friendly GUI, is written in R, and presents an example of a novel approach in data exploration. It is aimed at helping researchers interpret complex datasets, giving them ability to examine spatial information and genetic lineages step-by-step.

Central piece of the application is a map and an inputs panel that enables users to select samples from animals of interest. In case dataset contains a temporal component, filtering by date is also available. When spatial data is complemented with parentage data, functionality of the package is expanded with the ability to plot family pedigrees on-the-fly and suggestions of currently displayed animals' offspring.

Brief instructions on how to install and use the application are available at https://github. $\mathrm{com} / \mathrm{romunov} /$ wolfexplorer, where sample dataset from Monitoring of Conservation Status of Wolves in Slovenia in 2016/2017 (Bartol et al., 2017) is also supplied. Even though the name may indicate its inevitable connection to wolves, the application performs well with any dataset of this kind.

Wolfexplorer requires shinydashboard (Chang \& Borges Ribeiro, 2018), shinyjs (Attali, 2018), leaflet (Cheng, Karambelkar, \& Xie, 2018), RColorBrewer (Neuwirth, 2014), DT (Xie, 2018), sp (R. S. Bivand, Pebesma, \& Gomez-Rubio, 2013; E. J. Pebesma \& Bivand, 2005), rgdal (R. Bivand, Keitt, \& Rowlingson, 2018), data.table (Dowle \& Srinivasan, 2018), ggplot2 (Wickham, 2009), colourpicker (Attali, 2017), tidyr (Wickham \& Henry, 2018), plyr (Wickham, 2011), rgeos (R. Bivand \& Rundel, 2017), kinship2 (Therneau \& Sinnwell, 2015) and htmltools (RStudio \& Inc., 2017).

\section{Acknowledgements}

We thank Charlie Thompson for his contribution to the app. 


\section{Use}

Wolfexplorer has already been used for exploration of data gathered in different projects dealing with wolves in Slovenia, Croatia and Slovakia.

\section{References}

Attali, D. (2017). Colourpicker: A colour picker tool for shiny and for selecting colours in plots. Retrieved from https://CRAN.R-project.org/package=colourpicker

Attali, D. (2018). Shinyjs: Easily improve the user experience of your shiny apps in seconds. Retrieved from https://CRAN.R-project.org/package=shinyjs

Bartol, M., Černe, R., Hrovat, M., Jelenčič, M., Jonozovič, M., Kos, I., Krofel, M., et al. (2017). Monitoring of conservation status of wolves in slovenia in 2016/2017. Slovenian Forestry Service.

Bivand, R. S., Pebesma, E., \& Gomez-Rubio, V. (2013). Applied spatial data analysis with $R$, second edition. Springer, NY. Retrieved from http://www.asdar-book.org/

Bivand, R., \& Rundel, C. (2017). Rgeos: Interface to geometry engine - open source ('geos'). Retrieved from https://CRAN.R-project.org/package=rgeos

Bivand, R., Keitt, T., \& Rowlingson, B. (2018). Rgdal: Bindings for the 'geospatial' data abstraction library. Retrieved from https://CRAN.R-project.org/package=rgdal

Chang, W., \& Borges Ribeiro, B. (2018). Shinydashboard: Create dashboards with 'shiny'. Retrieved from https://CRAN.R-project.org/package=shinydashboard

Cheng, J., Karambelkar, B., \& Xie, Y. (2018). Leaflet: Create interactive web maps with the javascript 'leaflet' library. Retrieved from http://rstudio.github.io/leaflet/

Dowle, M., \& Srinivasan, A. (2018). Data.table: Extension of 'data.frame'. Retrieved from https://CRAN.R-project.org/package=data.table

Neuwirth, E. (2014). RColorBrewer: ColorBrewer palettes. Retrieved from https:// CRAN.R-project.org/package $=$ RColorBrewer

Pebesma, E. J., \& Bivand, R. S. (2005). Classes and methods for spatial data in R. $R$ News, 5(2), 9-13. Retrieved from https://CRAN.R-project.org/doc/Rnews/

RStudio, \& Inc. (2017). Htmltools: Tools for html. Retrieved from https://CRAN. R-project.org/package $=$ htmltools

Therneau, T. M., \& Sinnwell, J. (2015). Kinship2: Pedigree functions. Retrieved from https:/ /CRAN.R-project.org/package=kinship2

Wickham, H. (2009). Ggplot2: Elegant graphics for data analysis. Springer-Verlag New York. Retrieved from http://ggplot2.org

Wickham, H. (2011). The split-apply-combine strategy for data analysis. Journal of Statistical Software, 40(1), 1-29. doi:10.18637/jss.v040.i01

Wickham, H., \& Henry, L. (2018). Tidyr: Easily tidy data with 'spread()' and 'gather()' functions. Retrieved from https://CRAN.R-project.org/package=tidyr

Xie, Y. (2018). DT: A wrapper of the javascript library 'datatables'. Retrieved from https://CRAN.R-project.org/package=DT 